\title{
3CAC: improving the classification of phages and plasmids in metagenomic assemblies using assembly graphs
}

\author{
Lianrong $\mathrm{Pu}$ and Ron Shamir \\ Blavatnik School of Computer Science, Tel Aviv University \\ lianrongpu@mail.tau.ac.il, rshamir@tau.ac.il
}

\begin{abstract}
Bacteriophages and plasmids usually coexist with their host bacteria in microbial communities and play important roles in microbial evolution. Accurately identifying sequence contigs as phages, plasmids, and bacterial chromosomes in mixed metagenomic assemblies is critical for further unravelling their functions. Many classification tools have been developed for identifying either phages or plasmids in metagenomic assemblies. However, only two classifiers, PPR-Meta and viralVerify, were proposed to simultaneously identify phages and plasmids in mixed metagenomic assemblies. Due to the very high fraction of chromosome contigs in the assemblies, both tools achieve high precision in the classification of chromosomes but perform poorly in classifying phages and plasmids. Short contigs in these assemblies are often wrongly classified or classified as uncertain.

Here we present $3 \mathrm{CAC}$, a new three-class classifier that improves the precision of phage and plasmid classification. 3CAC starts with an initial three-class classification generated by existing classifiers and improves the classification of short contigs and contigs with low confidence classification by using proximity in the assembly graph. Evaluation on simulated metagenomes and on real human gut microbiome samples showed that 3CAC outperformed PPR-Meta and viralVerify in both precision and recall, and increased F1-score by 10-60 percentage points.
\end{abstract}

The 3CAC software is available on https://github.com/Shamir-Lab/3CAC.

Keywords: Metagenome · Three-class Classification · Assembly Graph - Phages · Plasmids. 


\section{Introduction}

The metagenomes of microbial communities are mainly composed of bacterial chromosomes and the associated extrachromosomal mobile genetic elements (eMGEs), such as plasmids and bacteriophages (phages). These eMGEs carry genes related to antibiotic resistance $[6,36,19]$, virulence factors $[14,30]$ and auxiliary metabolic pathways $[11,28,12]$. They can frequently move between species in the microbial community $[32,8]$ and enable their hosts to rapidly adapt to environmental changes [35,33]. Despite their important roles in horizontal gene transfer events and in antibiotic resistance, our understanding of these eMGEs is still limited. Part of the difficulty is the challenge of identifying such elements efficiently from mixed metagenomic assemblies [3, 16, 1, 2, 24, 34, 38].

Multiple algorithms have been developed for identifying either phages or plasmids from metagenomic assemblies in recent years. VirSorter and VirSorter2 identify viral metagenomic fragments by searching for reference homologs and testing enrichment of virus-like proteins $[29,10]$. These knowledge-based tools have high precision in virus classification but poor ability to identify novel viruses, due to reference database-associated bias. Other tools, such as DeepVirFinder [27], Seeker [4], and VIBRANT [12], use machine learning to learn k-mer signatures of viral sequences and perform better on novel virus classification, since they are more loosely linked to annotation databases. cBar is the first tool designed primarily for plasmid identification in metagenomes [40]. More recently, two supervised-learning approaches, PlasFlow [15] and PlasClass [23], were shown to classify plasmid fragments better from metagenomic assemblies. Although both phages and plasmids are commonly found in the metagenomes of microbial communities, all of these tools identify either only phages or only plasmids from metagenomic assemblies.

Currently, only two published tools, PPR-Meta [7] and viralVerify [2], can identify phages and plasmids simultaneously from metagenomic assemblies. However, due to the overwhelming abundance of chromosome fragments in the assemblies (usually $\geq 70 \%$ ), both tools achieve high precision in chromosome classification but very low precision in classification of phages and plasmids $[7,2]$. Moreover, classification of short contigs is challenging for all the existing classifiers, as they analyze each contig independently $[2,7,15,29,26]$. Here we present 3CAC (3-Class Adjacency based Classifier), an algorithm that employs existing two-class and three-class classifiers to generate an initial three-class classification with high precision, and then improves the classification of short contigs and of contigs classified with lower confidence by taking advantage of classification of their neighbors in the assembly graph. Evaluation on simulated and real metagenome datasets with short and long reads showed that 3CAC improved both precision and recall, and increased $\mathrm{F} 1$-score by at least 10 percentage points.

\section{Methods}

3CAC accepts as input a set of contigs and its associated assembly graph, uses the classification result of existing tools as a starting point, and repeatedly improves 
the classification using the assembly graph. Its output is a classification of each contig in the input as phage, plasmid, chromosome, or uncertain. The details of the algorithm are described below.

\subsection{Generating the initial classification}

3CAC exploits existing two-class and three-class classifiers to generate an initial three-class classification as follows.

(1) Generating a three-class classification. The algorithm runs either viralVerify or PPR-Meta on the set of the input contigs and classifies each contigs as phage, plasmid, chromosome, or uncertain. viralVerify was designed to classify contigs as viral, non-viral or uncertain. Moreover, for non-viral contigs, viralVerify can further classify them as plasmid or non-plasmid using $-p$ option. Here, we used - $p$ option of viralVerify to classify each of the input contigs as viral, plasmid, chromosome, or uncertain. PPR-Meta calculates three scores representing the probabilities of a contig to be classified as a phage, plasmid, or chromosome. By default, PPR-Meta classifies a contig into the class with the highest score. If a specified score threshold is provided and no score passes the threshold, the sequence will be classified as uncertain. Here, we ran PPR-Meta with a score threshold of 0.7 .

(2) Improving plasmid classification. To improve the precision of plasmid classification, PlasClass is run on contigs classified as plasmids in step (1). PlasClass outputs for each contig the probability that it originated from a plasmid. By default, PlasClass classifies a contig as plasmid if it has a probability $>0.5$ and as chromosome otherwise. To assure high precision, here we identify contigs with probability $\geq 0.7$ as plasmids. Contigs with probability $\leq 0.3$ are moved to the chromosome class. The remaining contigs are reclassified as uncertain.

(3) Improving phage classification. Similarly, in order to improve the precision of phage classification, we run DeepVirFinder on all contigs classified as phages in step (1). DeepVirFinder generates a score and a p-value for each input contig. Contigs with higher scores or lower p-values are more likely to be viral sequences. Here, a contig is kept in the phage class if its p-value $\leq 0.03$ and moved to the chromosome class if its $\mathrm{p}$-value $>0.03$ and its score $\leq 0.5$. The remaining contigs are reclassified as uncertain.

We will denote the algorithm up to this step Initial(vV) and Initial(PM) if viralVerify or PPR-Meta were used in step (1), respectively.

\subsection{Refining the classification using the assembly graph}

In genomics and metagenomics, assembly graphs, such as de Bruijn graphs [18, $25]$ and string graphs [21,31], are used as the core data structure to combine overlapped reads (or k-mers) into contigs. Initially, nodes are $k$-mers and edges represent $(k+1)$-long overlaps between them. Each longest path of nodes with indegree and outdegree $=1$ is then collapsed into a single node representing the corresponding sequence contig. In our description below, nodes in the graph are 
the contigs, and the neighbors of a contig are its adjacent nodes. Existing classifiers take contigs as input and classify each of them independently based on its sequence. The overlap information between neighboring contigs in the assembly graphs was ignored by all the existing classifiers. However, recent studies showed that neighboring contigs in an assembly graph are more likely to come from the same taxonomic group [5,20]. Based on this insight, here we exploit the assembly graph to improve the classification by the following two steps.

(1) Correction of classified contigs. A classified contig is called incongruous if it has $\geq 2$ classified neighbors and all of them belong to same class, while this contig belongs to a different class. We reason that an incongruous contig was wrongly classified and its classification needs to be consistent with its classified neighbors. Therefore, $3 \mathrm{CAC}$ scans all the incongruous contigs in decreasing order of the number of their classified neighbors and corrects the classification of each incongruous contig to match its classified neighbors. Note that once an incongruous contig is corrected, this contig and all its neighbors will not be corrected anymore.

(2) Propagation from classified contigs to unclassified contigs. An unclassified contig is called implied if it has one or more classified neighbors and all of them belong to same class. 3CAC dynamically maintains a sorted list of implied contigs in decreasing order of the number of their classified neighbors. At each iteration, 3CAC classifies the first implied contig on the list according to its classified neighbors and then updates the sorted list. Note that only the unclassified neighbors of the first implied contig need to be updated at each iteration. We repeat this step until the list is empty.

Figure 1 shows the result of applying steps (1) and (2) in a small assembly graph, which is part of the graph generated by assembling simulated long reads (Sim4; see details in the Results section).

We will use the names $\mathbf{3 C A C}(\mathbf{v V})$ and $\mathbf{3 C A C}(\mathbf{P M})$ for the full $3 \mathrm{CAC}$ algorithms initialized with viralVerify and PPR-Meta solutions, respectively.

(a) Initial (vV)

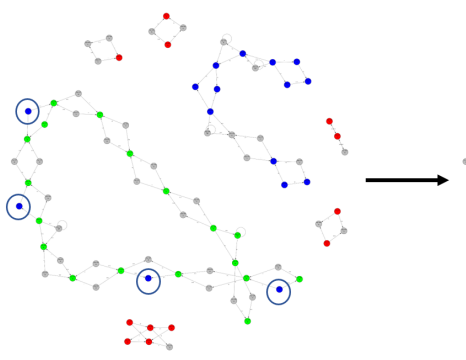

(b) After correction

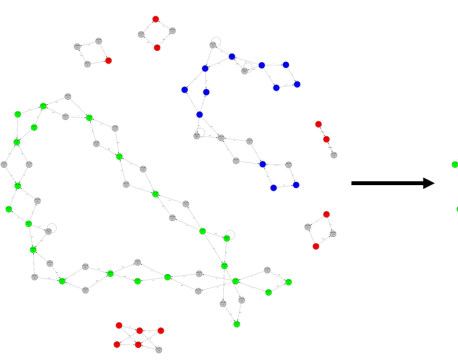

(c) After propagation

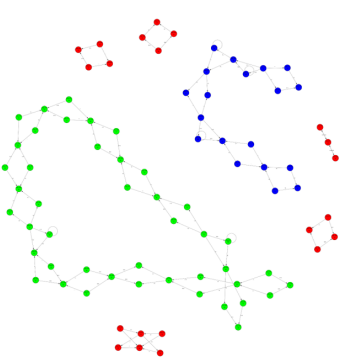

Fig. 1. An example of improving the classification using the assembly graph. Vertices with color red, blue, green, and grey represent contigs classified as phages, plasmids, chromosomes, and uncertain, respectively. (a) The result of Initial(vV). (b) After the correction step. The four contigs encircled in (a) were corrected. (c) After the propagation step. 


\section{Results}

We tested 3CAC on both simulated and real metagenome assemblies and compared it to PPR-Meta and viralVerify.

\subsection{Evaluation criteria}

3CAC, viralVerify and PPR-Meta were evaluated based on precision, recall, and F1 score, calculated as follows.

- Precision: the fraction of correctly classified contigs among all classified contigs. Note that uncertain contigs were not included in the calculation.

- Recall: the fraction of correctly classified contigs among all contigs.

- F1 score: the harmonic mean of the precision and recall, which can be calculated as: $F 1$ score $=(2 *$ precision $*$ recall $) /($ precision + recall $)$.

Following $[23,7]$, the precision, recall, and F1 score here were calculated by counting the number of contigs and did not take into account their length. The precision and recall were also calculated separately for phage, plasmid and chromosome classification. For example, the precision of phage classification was calculated as the fraction of correctly classified phage contigs among all contigs classified as phages, and the recall of phage classification was calculated as the fraction of correctly classified phage contigs among all phage contigs.

\subsection{Performance on simulated metagenome assemblies}

We generated two short-read and two long-read metagenome assemblies as follows. Sequences of complete bacterial genomes were randomly selected from the NCBI database along with their associated plasmids. The abundance of bacterial genomes was modeled by the log-normal distribution and the copy numbers of plasmids were simulated by the geometric distribution as in [23]. The phage genomes and their abundance profiles were sampled from [26]. Two metagenomic datasets of different complexities were designed. For each of the datasets, 150bp-long short reads were simulated from the genome sequences using InSilicoSeq [9] and assembled by metaSPAdes [22]. Long reads were simulated from the genome sequences using NanoSim [39] and assembled by metaFlye [13]. The error rate of long reads was $9.8 \%$ and their average length was $14.9 \mathrm{~kb}$. For each assembly, contigs were matched to the reference genomes used in the simulation by minimap2 [17]. Contigs having matches to a reference genome with $\geq 90 \%$ mapping identity along $\geq 80 \%$ of the contig length were assigned to the class of that reference, and these assignments were used as the gold standard to test the classifiers. Table 1 presents a summary of the simulated metagenome assemblies.

Figure 2 shows the performance of PPR-Meta, viralVerify and the first phase of 3CAC on these simulated metagenome assemblies. Both PPR-Meta and viralVerify had high precision in chromosome classification, but their precision in phage and plasmid classification was usually low. Further analysis revealed that 
Table 1. Properties of the simulated and the real metagenome datasets and of their assemblies. The number of genome references for the real human gut metagenomes is the number of all complete chromosome, plasmid and phage genomes in NCBI database.

\begin{tabular}{|c|c|c|c|c|c|c|c|c|c|}
\hline & \multirow{2}{*}{ Read type } & \multirow{2}{*}{$\begin{array}{c}\text { Number of } \\
\text { reads }\end{array}$} & \multicolumn{3}{|c|}{ \# of genome references } & \multicolumn{3}{|c|}{ \# of assembled contigs } & \multirow{2}{*}{$\begin{array}{l}\text { Short contigs } \\
\qquad(<1 \mathrm{~kb})\end{array}$} \\
\hline & & & chromosome & plasmid & phage & chromosom & plasmic & phage & \\
\hline Sim1 & MiSeq & \begin{tabular}{|c|c}
$61 \mathrm{M}$ \\
\end{tabular} & 50 & 193 & 200 & 12,494 & 1,699 & 696 & 8,991 \\
\hline $\operatorname{Sim} 2$ & MiSeq & $100 \mathrm{M}$ & 100 & 410 & 500 & 40,412 & 5,350 & 2,926 & 33,640 \\
\hline Sim3 & Nanopore & $0.5 \mathrm{M}$ & 50 & 193 & 200 & 890 & 166 & 175 & 45 \\
\hline $\operatorname{Sim} 4$ & Nanopore & $1 \mathrm{M}$ & 100 & 410 & 500 & 2,491 & 395 & 413 & 152 \\
\hline Sim2021-Miseq & MiSeq & $100 \mathrm{M}$ & 100 & 433 & 500 & 34,211 & 3,512 & 2,014 & 25,861 \\
\hline Sim2021-Nano & Nanopore & $1 \mathrm{M}$ & 100 & 433 & 500 & 2,413 & 345 & 342 & 143 \\
\hline Gut-HiSeq & HiSeq & $53.8 \mathrm{M}$ & 19,053 & 20,838 & 13,903 & 130,252 & 943 & 383 & 110,128 \\
\hline Gut-Pacbio & Pacbio & $14.7 \mathrm{M}$ & 19,053 & 20,838 & 13,903 & 4,671 & 64 & 8 & 723 \\
\hline
\end{tabular}

both of the algorithms distinguished well between phages and plasmids. Their low precision in phage and plasmid classification was due to contamination from chromosome contigs (Supplementary Table A.1). Utilizing two-class classifiers, PlasClass and DeepVirFinder, the first phase of 3CAC improved markedly the precision in phage and plasmid classification, while it decreased a little bit the precision in chromosome classification (Figure 2, Supplementary Table A.2). In contrast, recall decreased in phage and plasmid classification, but increased in chromosome classification (Supplementary Figure B.1).

Figure 3 shows the results of initial phase of $3 \mathrm{CAC}$ on the short-read simulated metagenome assemblies for different contig lengths. Short contigs tended to have lower recall in the initial classification of $3 \mathrm{CAC}$, while precision was not sensitive to the contig length. When the initial classification of 3CAC was generated based on PPR-Meta solution, recall decreased sharply for contigs with length $<$ $1 \mathrm{~kb}$. When viralVerify solution was used, recall was even lower for contig shorter than $1 \mathrm{~kb}$ and improvement with size was roughly linear. We reasoned that these classifiers classified each of the input contigs independently, and so short contigs could not be classified reliably. However, Table 1 shows that more than half of the contigs assembled from short reads are shorter than $1 \mathrm{~kb}$. To assist in the classification of these short contigs, 3CAC was designed to take advantage of the longer contigs with confident classification and that are neighbors of these short contigs in the assembly graph. Figure 3 shows that 3CAC significantly increased recall for all contigs with almost no loss of precision. Remarkably, the recall for contigs shorter than $1 \mathrm{~kb}$ increased from $<0.2$ to $\geq 0.8$. For contigs assembled from long reads, $3 \mathrm{CAC}$ not only improved the recall substantially but also slightly improved the precision (Figure 4).

The analysis above shows that the two phases of 3CAC algorithm improved the precision and recall for the three-class classification. Evaluation of PPRMeta, viralVerify and 3CAC on these simulated metagenome assemblies showed that 3CAC performed the best in both precision and recall (Figure 5, Supplementary Table A.1, A.3). We also calculated the precision, recall and F1 scores for phage, plasmid, and chromosome classification separately (Supplementary Table A.4). 3CAC had the best F1 scores on all the datasets. Note that PPR-Meta here was run with default setting. Running PPR-Meta with 0.7 score thresh- 

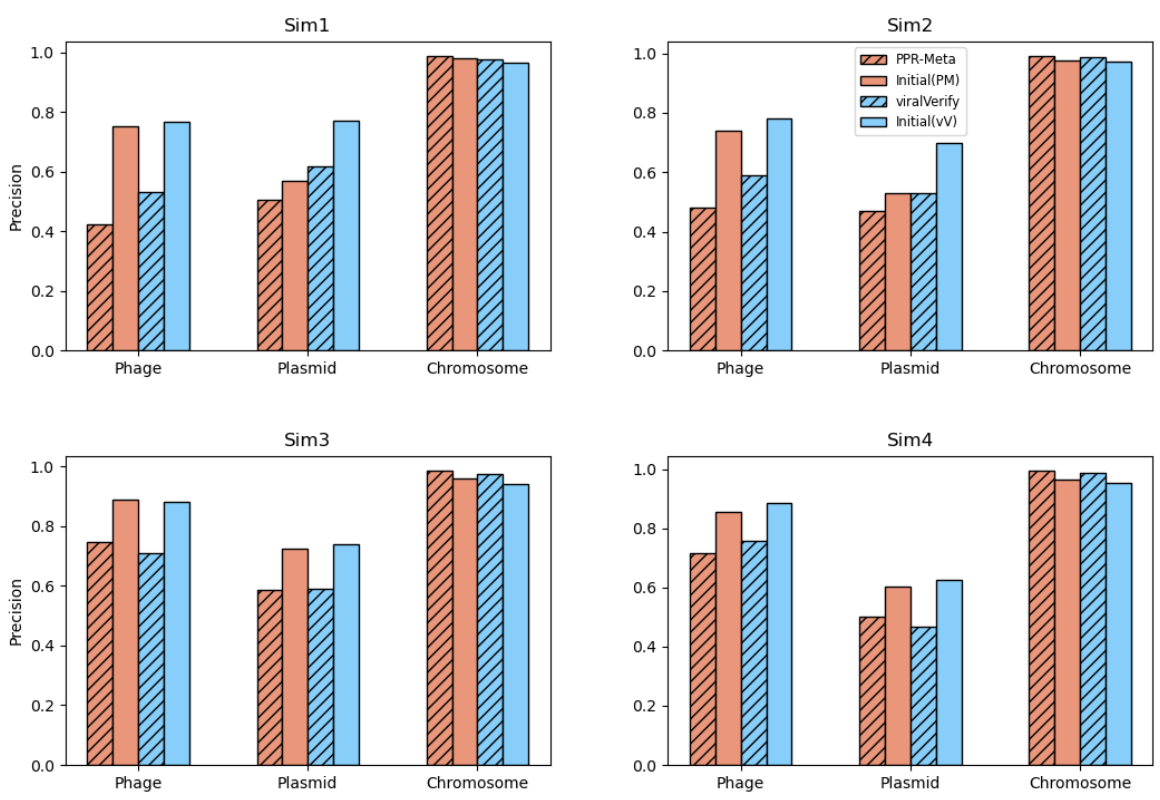

Fig. 2. Precision of the initial classification of 3CAC compared to PPR-Meta and viralVerify. Sim1 and Sim2 are assembled from short reads. Sim3 and Sim4 are assembled from long reads. See supplementary Figure B.1 for recall.
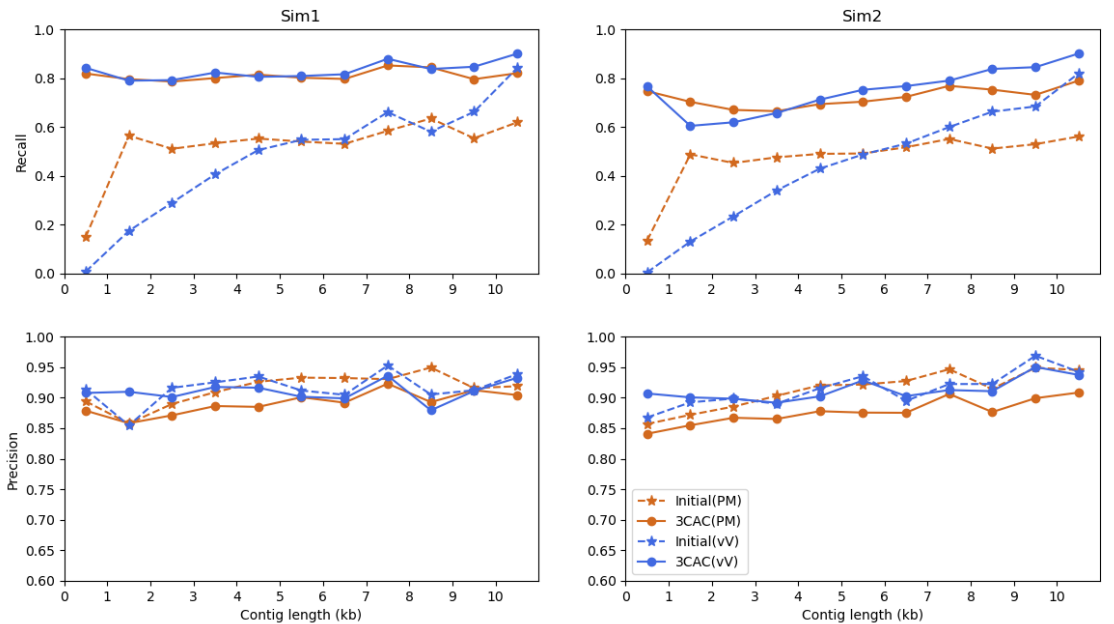

Fig. 3. Performance on contigs assembled from simulated short reads. Results are shown for contigs of lengths $<1 \mathrm{~kb}, 1-2 \mathrm{~kb}, \ldots, 9-10 \mathrm{~kb}, \geq 10 \mathrm{~kb}$. 

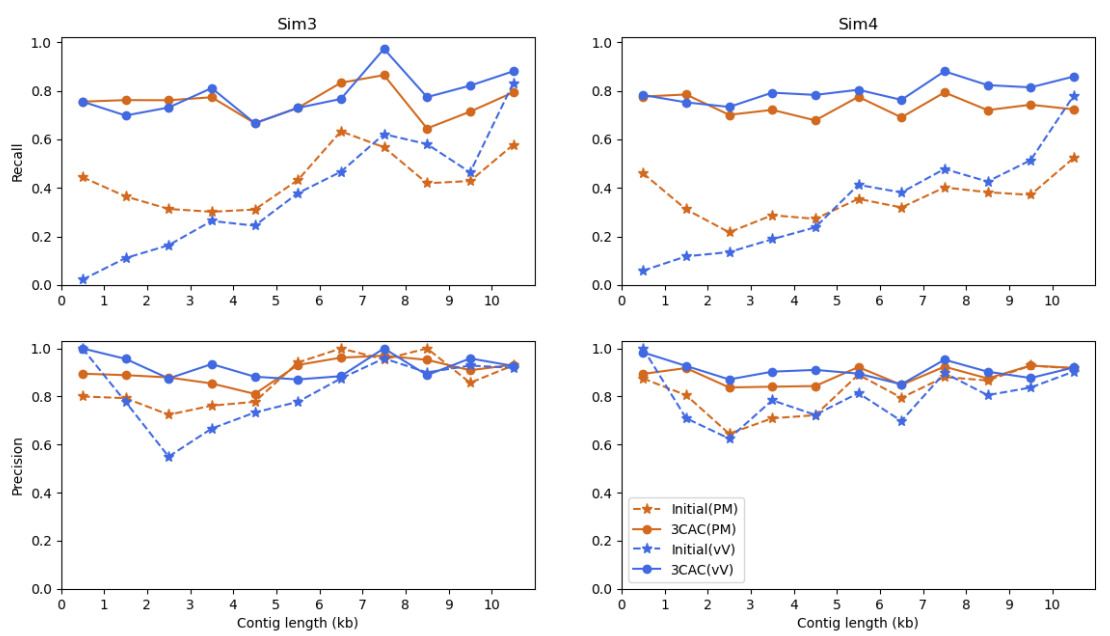

Fig. 4. Performance on contigs assembled from simulated long reads. Results are shown for contigs of lengths $<1 \mathrm{~kb}, 1-2 \mathrm{~kb}, \ldots, 9-10 \mathrm{~kb}, \geq 10 \mathrm{~kb}$.

old (as done in Initial(PM)) resulted in higher precision but lower recall and lower F1 score. Supplementary Table A.5 shows that 3CAC also outperformed PPR-Meta with 0.7 score threshold.

A potential bias in evaluating $3 \mathrm{CAC}$ may be created by the fact that the simulated datasets contain sequences that may have been used for training the various classifiers. To evaluate the ability of 3CAC to classify novel species, additional short-read and long-read metagenome simulations were designed by selecting genomes released on NCBI after January 2021 (Sim2021-Miseq and Sim2021-Nano in Table 1). Since all the classifiers that we used were developed prior to that date, all the tested genomes in these metagenome assemblies were not included in the training of the classifiers used by 3CAC. Evaluation on Sim2021-Miseq and Sim2021-Nano showed that 3CAC performed equally well in classification of novel species (Supplementary Figure B.2 and Table A.5).

Overall $3 \mathrm{CAC}(\mathrm{vV})$ achieved best precision, recall, and $\mathrm{F} 1$ score in all six simulations, and 3CAC(PM) was a close second (Supplementary Table A.5). PPR-Meta and viralVerify had 25-60 lower percentage points in F1 score on the short-read simulations, and 14-30 lower percentage points on the long-read simulations.

\subsection{Performance on human gut microbiome samples}

Five publicly available human gut microbiome samples with short-read sequencing datasets (NCBI accession numbers: ERR12976697, ERR1297651, ERR1297751, ERR1297845, ERR1297770) were selected and assembled together using metaSPAdes [22]. Another set of five human gut microbiome samples with long-read sequencing datasets (NCBI accessions: SRX2529348, SRX2529347, SRX2529346, SRX2529341, SRX2529340) were selected from [34] and assembled together us- 

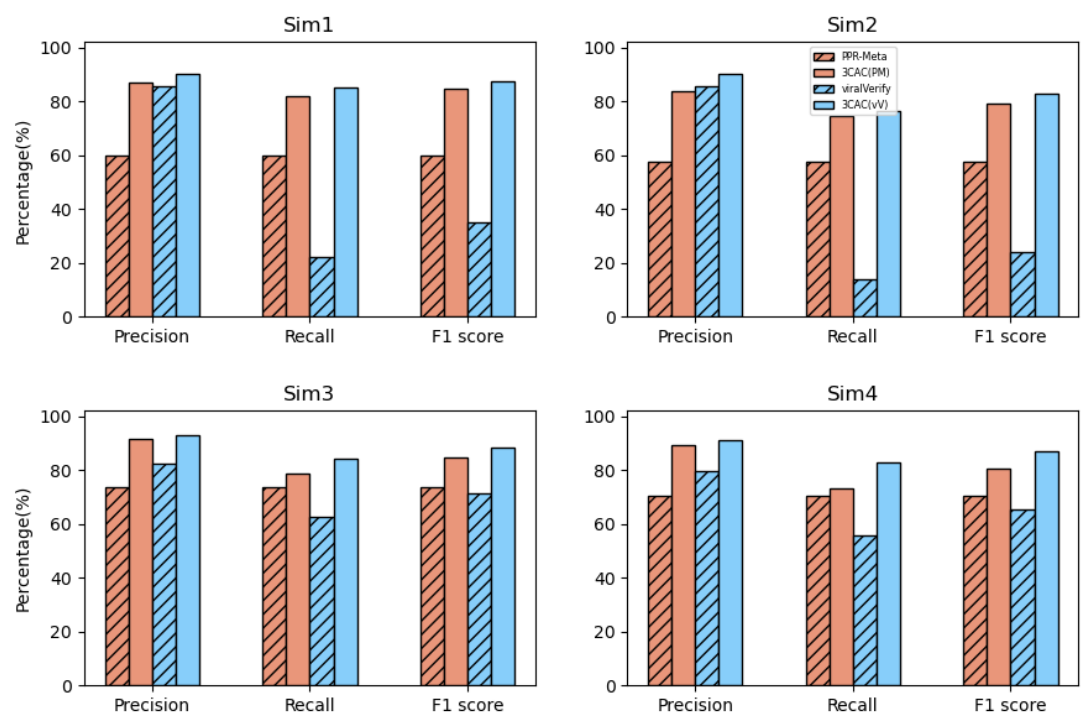

Fig. 5. Performance of three-class classifiers on the simulated metagenome assemblies. Sim1 and Sim2 are assembled from short reads. Sim3 and Sim4 are assembled from long reads.

ing metaFlye [13]. To identify the class of contigs in the real metagenome assemblies, we downloaded all complete phage, plasmid and chromosome genomes from NCBI database and mapped contigs to all the reference genomes using minimap2 [17]. A contig was considered matched to a reference sequence if it had $\geq$ $80 \%$ mapping identity along $\geq 80 \%$ of the contig length. Contigs that matched to reference genomes of two or more classes were excluded to avoid ambiguity. Overall, 131,578 out of 469,022 contigs in the short-read assembly and 4,743 out of 12,541 contigs in the long-read assembly had matches to a single class and were used as the gold standard to test the classifiers. Table 1 summarizes the properties of the datasets and the assemblies.

Figures 6(a) and 7(a) show the results of PPR-Meta, viralVerify and 3CAC on the short-read and long-read assemblies, respectively. On the long-read assembly, $3 \mathrm{CAC}(\mathrm{vV})$ and $3 \mathrm{CAC}(\mathrm{PM})$ had comparable performance. 3CAC was best in precision, recall and F1 score (Figure 7).

Interestingly, on the short-read assembly, 3CAC(PM) and PPR-Meta had higher $\mathrm{F} 1$ score than $3 \mathrm{CAC}(\mathrm{vV})$ (Figure 6 (a)). Further analysis revealed that this was due to a large number of isolated contigs in the short-read assembly graph. The second phase of $3 \mathrm{CAC}$ was only performed on contigs that have neighbours in the assembly graph. However, $59 \%$ of the contigs assembled from short reads were isolated and had no neighbors in the assembly graph, while the fraction on the long-read assembly was only $21 \%$. Figures 6 (b) and 7 (b) show the results on the non-isolated contigs in the assembly graph. For both long- 

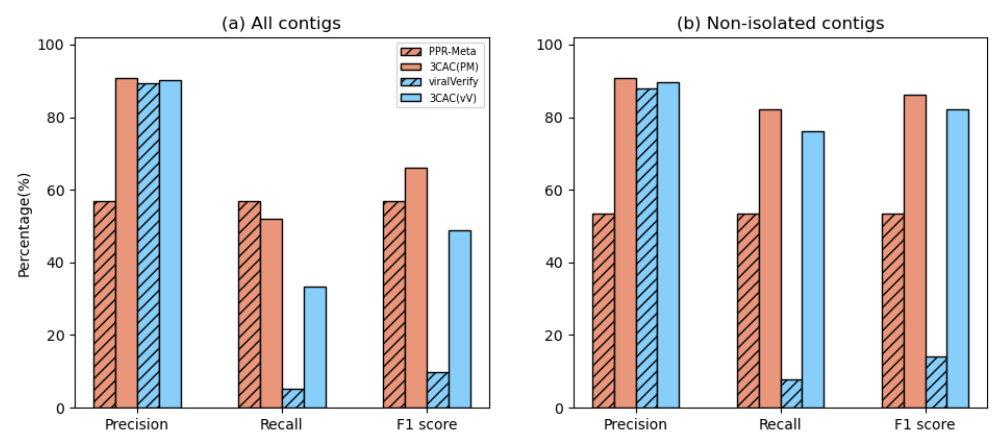

Fig. 6. Performance of three-class classifiers on contigs assembled from short-read sequencing of human gut microbiome samples. (a) performance on all contigs; (b) performance on non-isolated contigs in the assembly graph.

read and short-read assemblies, 3CAC(PM) and 3CAC(vV) had comparable performance and outperformed PPR-meta and viralVerify in precision, recall, and F1 score.

Supplementary Table A.4 shows the precision, recall and F1 score separately for phage, plasmid and chromosome classification in both short-read and longread assemblies. Compared to PPR-Meta, 3CAC had higher precision in classification of phages and plasmids, at the cost of lower recall and tended to have better F1 scores. In chromosome classification, 3CAC performed the best in the long-read assembly while PPR-Meta performed slightly better in the short-read assembly.
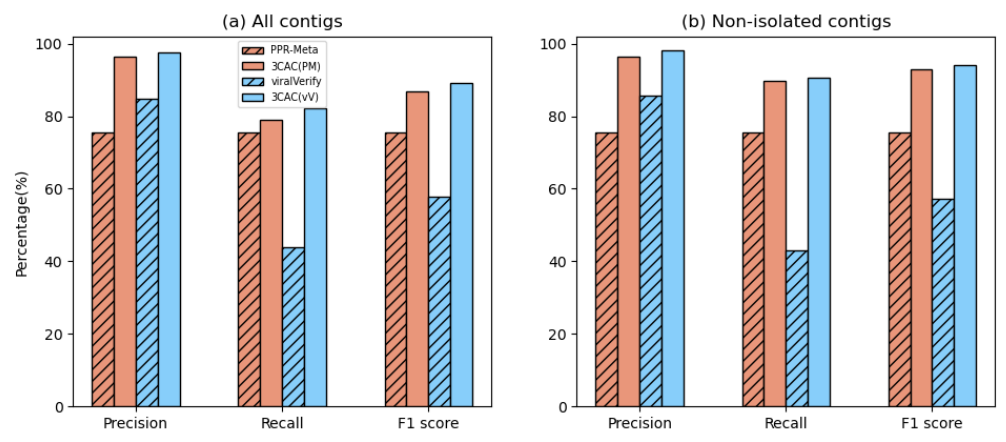

Fig. 7. Performance of three-class classifiers on contigs assembled from longread sequencing of human gut microbiome samples. (a) performance on all contigs; (b) performance on non-isolated contigs in the assembly graph.

\subsection{Software and Resource usage}

3CAC uses classification results generated by existing classifiers as a starting point, and so the running time of its first phase depends on the classifiers used. 
Supplementary Table A.6 shows that viralVerify was most time consuming, followed by PPR-Meta. PlasClass and DeepVirFinder were fast as they were only run on a subset of the input contigs. Time consumed by the second phase of 3CAC was tiny compared to PPR-Meta and viralVerify. Performance was measured on a 44-core, $2.2 \mathrm{GHz}$ server with $792 \mathrm{~GB}$ of RAM. 3CAC is freely available on Github via https://github.com/Shamir-Lab/3CAC.

\section{Discussion and Conclusion}

In this study we introduced 3CAC, a new three-class classifier to classify contigs in assembly graphs into bacterial, plasmid, viral, and uncertain. 3CAC builds on initial three-class classifications generated by viralVerify or PPR-Meta and improves the classification of short and uncertain contigs by exploiting the structure of the assembly graph. Evaluation on real and simulated metagenomes assembled from both short and long reads showed that 3CAC significantly improved the recall with almost no loss of the precision.

In the correction step of $3 \mathrm{CAC}$, the order of treating incongruous contigs with the same number of classified neighbors may affect the results. A similar situation occurs in the propagation step. We tried several random orders and the results were very stable $(<1 \%$ difference, Supplementary Table A.7).

Should a user interested specifically in a two-way classification prefer a dedicated two-way classifier over 3CAC? We compared PlasClass to 3CAC in plasmid classification, and DeepVirFinder to 3CAC in phage classification. The results on the six simulated datasets show that 3CAC had superior precision and F1 score in all cases (Supplementary Table A.8 and Table A.9).

Evaluation the performance of classifiers on real metagenome assemblies remains challenging due to the lack of gold standard. By mapping contigs to all the available reference genomes, we are able to identify the class of a fraction of the contigs. However, as shown in previous studies [7], some plasmid genomes are quite similar to their host bacterial chromosomes. Thus, many contigs from metagenome assemblies have matches to both plasmid and chromosome reference genomes, and it is hard to identify their classes. Additionally, many contigs with no matches to the reference database may represent novel species, but they were excluded from our evaluation. Keeping in mind these shortcomings of the gold standard for real metagenome assemblies, 3CAC outperformed existing three-class classifiers substantially.

3CAC has some limitations. The propagation step of 3CAC can greatly improve the recall, but it can only be performed on non-isolated contigs in the assembly graph. The recall of isolated contigs is still limited by the performance of existing classifiers. 3CAC relies on existing two-class and three-class classifiers. In the future, we plan to extend $3 \mathrm{CAC}$ to a stand-alone classification tool. The detection of prophages and other non-bacterial sequences that integrate into bacterial genome is challenging to all classifiers, including 3CAC. Finally, there is room for extending $3 \mathrm{CAC}$ to a four-class algorithm that would be able to classify also eukaryotic contigs in metagenome assemblies [37]. 


\section{Acknowledgments}

This study was supported in part by grant 2016694 from the United State - Israel Binational Science Foundation (BSF), Jerusalem, Israel and the United States National Science Foundation (NSF). L.P. was supported in part by a fellowship from the Edmond J. Safra Center for Bioinformatics at Tel-Aviv University. L.P. was also supported in part by postdoctoral fellowships from the Planning and Budgeting Committee (PBC) of the Council for Higher Education (CHE) in Israel.

\section{References}

1. Antipov, D., Raiko, M., Lapidus, A., Pevzner, P.A.: Plasmid detection and assembly in genomic and metagenomic data sets. Genome Research 29(6), 961-968 (2019)

2. Antipov, D., Raiko, M., Lapidus, A., Pevzner, P.A.: Metaviral SPAdes: assembly of viruses from metagenomic data. Bioinformatics 36(14), 4126-4129 (2020)

3. Arredondo-Alonso, S., Willems, R.J., Van Schaik, W., Schürch, A.C.: On the (im) possibility of reconstructing plasmids from whole-genome short-read sequencing data. Microbial Genomics 3(10) (2017)

4. Auslander, N., Gussow, A.B., Benler, S., Wolf, Y.I., Koonin, E.V.: Seeker: alignment-free identification of bacteriophage genomes by deep learning. Nucleic Acids Research 48(21), e121-e121 (2020)

5. Barnum, T.P., Figueroa, I.A., Carlström, C.I., Lucas, L.N., Engelbrektson, A.L., Coates, J.D.: Genome-resolved metagenomics identifies genetic mobility, metabolic interactions, and unexpected diversity in perchlorate-reducing communities. The ISME Journal 12(6), 1568-1581 (2018)

6. Calero-Cáceres, W., Ye, M., Balcázar, J.L.: Bacteriophages as environmental reservoirs of antibiotic resistance. Trends in Microbiology 27(7), 570-577 (2019)

7. Fang, Z., Tan, J., Wu, S., Li, M., Xu, C., Xie, Z., Zhu, H.: PPR-Meta: a tool for identifying phages and plasmids from metagenomic fragments using deep learning. GigaScience 8(6), giz066 (2019)

8. Frost, L.S., Leplae, R., Summers, A.O., Toussaint, A.: Mobile genetic elements: the agents of open source evolution. Nature Reviews Microbiology 3(9), 722-732 (2005)

9. Gourlé, H., Karlsson-Lindsjö, O., Hayer, J., Bongcam-Rudloff, E.: Simulating illumina metagenomic data with insilicoseq. Bioinformatics 35(3), 521-522 (2019)

10. Guo, J., Bolduc, B., Zayed, A.A., Varsani, A., Dominguez-Huerta, G., Delmont, T.O., Pratama, A.A., Gazitúa, M.C., Vik, D., Sullivan, M.B., et al.: Virsorter2: a multi-classifier, expert-guided approach to detect diverse DNA and RNA viruses. Microbiome 9(1), 1-13 (2021)

11. Hurwitz, B.L., U'Ren, J.M.: Viral metabolic reprogramming in marine ecosystems. Current Opinion in Microbiology 31, 161-168 (2016)

12. Kieft, K., Zhou, Z., Anantharaman, K.: Vibrant: automated recovery, annotation and curation of microbial viruses, and evaluation of viral community function from genomic sequences. Microbiome 8(1), 1-23 (2020)

13. Kolmogorov, M., Bickhart, D.M., Behsaz, B., Gurevich, A., Rayko, M., Shin, S.B., Kuhn, K., Yuan, J., Polevikov, E., Smith, T.P., et al.: metaFlye: scalable long-read metagenome assembly using repeat graphs. Nature Methods 17(11), 1103-1110 (2020) 
14. Kraushaar, B., Hammerl, J., Kienol, M., Heinig, M., Sperling, N., Dinh Thanh, M., Reetz, J., Jackel, C., Fetsch, A., Hertwig, S.: Acquisition of virulence factors in livestock-associated mrsa: lysogenic conversion of cc398 strains by virulence gene-containing phages. Sci Rep 7: 2004 (2017)

15. Krawczyk, P.S., Lipinski, L., Dziembowski, A.: Plasflow: predicting plasmid sequences in metagenomic data using genome signatures. Nucleic Acids Research 46(6), e35-e35 (2018)

16. Krishnamurthy, S.R., Wang, D.: Origins and challenges of viral dark matter. Virus Research 239, 136-142 (2017)

17. Li, H.: Minimap2: pairwise alignment for nucleotide sequences. Bioinformatics 34(18), 3094-3100 (2018)

18. Lin, Y., Yuan, J., Kolmogorov, M., Shen, M.W., Chaisson, M., Pevzner, P.A.: Assembly of long error-prone reads using de Bruijn graphs. Proceedings of the National Academy of Sciences 113(52), E8396-E8405 (2016)

19. Lopatkin, A., Meredith, H., Srimani, J., Pfeiffer, C., Durrett, R., You, L.: Persistence and reversal of plasmid-mediated antibiotic resistance. Nature Communications 8: 1689 (2017)

20. Mallawaarachchi, V., Wickramarachchi, A., Lin, Y.: Graphbin: refined binning of metagenomic contigs using assembly graphs. Bioinformatics 36(11), 3307-3313 (2020)

21. Myers, E.W.: The fragment assembly string graph. Bioinformatics 21(suppl_2), ii79-ii85 (2005)

22. Nurk, S., Meleshko, D., Korobeynikov, A., Pevzner, P.: metaSPAdes: a new versatile de novo metagenomics assembler. arXiv preprint arXiv:1604.03071 (2016)

23. Pellow, D., Mizrahi, I., Shamir, R.: Plasclass improves plasmid sequence classification. PLoS Computational Biology 16(4), e1007781 (2020)

24. Pellow, D., Zorea, A., Probst, M., Furman, O., Segal, A., Mizrahi, I., Shamir, R.: Scapp: An algorithm for improved plasmid assembly in metagenomes. Microbiome 9(1), 1-12 (2021)

25. Pevzner, P.A., Tang, H., Waterman, M.S.: An Eulerian path approach to DNA fragment assembly. Proceedings of the National Academy of Sciences 98(17), 97489753 (2001)

26. Ren, J., Ahlgren, N.A., Lu, Y.Y., Fuhrman, J.A., Sun, F.: Virfinder: a novel kmer based tool for identifying viral sequences from assembled metagenomic data. Microbiome 5(1), 1-20 (2017)

27. Ren, J., Song, K., Deng, C., Ahlgren, N.A., Fuhrman, J.A., Li, Y., Xie, X., Poplin, R., Sun, F.: Identifying viruses from metagenomic data using deep learning. Quantitative Biology pp. 1-14 (2020)

28. Rosenwasser, S., Ziv, C., Van Creveld, S.G., Vardi, A.: Virocell metabolism: metabolic innovations during host-virus interactions in the ocean. Trends in Microbiology 24(10), 821-832 (2016)

29. Roux, S., Enault, F., Hurwitz, B.L., Sullivan, M.B.: Virsorter: mining viral signal from microbial genomic data. PeerJ 3, e985 (2015)

30. Sarowska, J., Futoma-Koloch, B., Jama-Kmiecik, A., Frej-Madrzak, M., Ksiazczyk, M., Bugla-Ploskonska, G., Choroszy-Krol, I.: Virulence factors, prevalence and potential transmission of extraintestinal pathogenic escherichia coli isolated from different sources: recent reports. Gut Pathogens 11(1), 1-16 (2019)

31. Simpson, J.T., Durbin, R.: Efficient de novo assembly of large genomes using compressed data structures. Genome Research 22(3), 549-556 (2012) 
32. Sitaraman, R.: Prokaryotic horizontal gene transfer within the human holobiont: ecological-evolutionary inferences, implications and possibilities. Microbiome 6(1), 1-14 (2018)

33. Smalla, K., Jechalke, S., Top, E.M.: Plasmid detection, characterization, and ecology. Microbiology Spectrum 3(1), 3-1 (2015)

34. Suzuki, Y., Nishijima, S., Furuta, Y., Yoshimura, J., Suda, W., Oshima, K., Hattori, M., Morishita, S.: Long-read metagenomic exploration of extrachromosomal mobile genetic elements in the human gut. Microbiome 7(1), 1-16 (2019)

35. Thomas, C.M., Nielsen, K.M.: Mechanisms of, and barriers to, horizontal gene transfer between bacteria. Nature Reviews Microbiology 3(9), 711-721 (2005)

36. Wein, T., Hülter, N., Mizrahi, I., Dagan, T.: Emergence of plasmid stability under non-selective conditions maintains antibiotic resistance. Nature Communications 10: 2595 (2019)

37. West, P.T., Probst, A.J., Grigoriev, I.V., Thomas, B.C., Banfield, J.F.: Genomereconstruction for eukaryotes from complex natural microbial communities. Genome Research 28(4), 569-580 (2018)

38. Yahara, K., Suzuki, M., Hirabayashi, A., Suda, W., Hattori, M., Suzuki, Y., Okazaki, Y.: Long-read metagenomics using promethion uncovers oral bacteriophages and their interaction with host bacteria. Nature Communications 12(1), 1-12 (2021)

39. Yang, C., Chu, J., Warren, R.L., Birol, I.: Nanosim: nanopore sequence read simulator based on statistical characterization. GigaScience 6(4), gix010 (2017)

40. Zhou, F., Xu, Y.: cBar: a computer program to distinguish plasmid-derived from chromosome-derived sequence fragments in metagenomics data. Bioinformatics 26(16), 2051-2052 (2010) 
bioRxiv preprint doi: https://doi.org/10.1101/2021.11.05.467408; this version posted January 26, 2022. The copyright holder for this preprint (which was not certified by peer review) is the author/funder, who has granted bioRxiv a license to display the preprint in perpetuity. It is made available under aCC-BY-ND 4.0 International license.

\section{Supplementary Material}

\section{A Supplementary tables}

Table A.1. Performance of viralVerify and PPR-Meta on simulated metagenome assemblies. PPR-Meta was run with 0.7 score threshold to assure high precision.

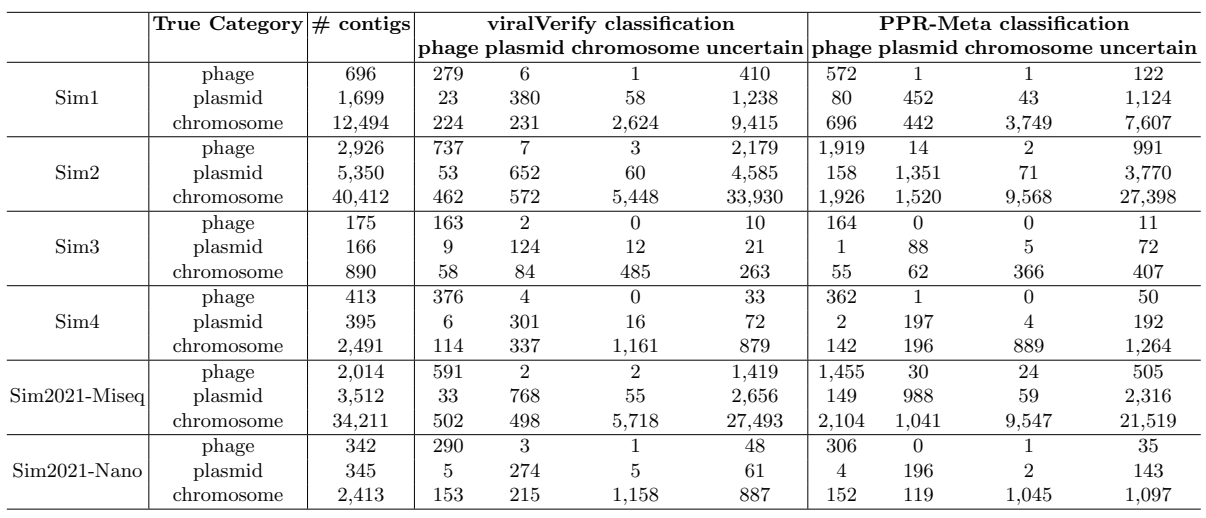

Table A.2. Performance of Initial(vV) and Initial(PM) on simulated metagenome assemblies.

\begin{tabular}{c|c|c|cccc|cccc}
\hline & True Category & \# contigs & \multicolumn{4}{c|}{ Initial(vV) } & \multicolumn{4}{c}{ Initial(PM) } \\
& & & phage plasmid chromosome & uncertain & phage plasmid chromosome uncertain \\
\hline \multirow{3}{*}{ Sim1 } & phage & 696 & 249 & 3 & 8 & 436 & 442 & 0 & 8 & 246 \\
& plasmid & 1,699 & 7 & 310 & 88 & 1,294 & 10 & 372 & 75 & 1,242 \\
& chromosome & 12,494 & 69 & 90 & 2,797 & 9,538 & 137 & 284 & 3,936 & 8,137 \\
\hline \multirow{3}{*}{ Sim2 } & phage & 2,926 & 653 & 4 & 35 & 2,234 & 1,136 & 9 & 45 & 1,736 \\
& plasmid & 5,350 & 18 & 499 & 132 & 4,701 & 33 & 964 & 213 & 4,140 \\
& chromosome & 40,412 & 165 & 213 & 5,803 & 34,231 & 363 & 842 & 10,092 & 29,115 \\
\hline \multirow{3}{*}{ Sim3 } & phage & 175 & 139 & 1 & 11 & 24 & 145 & 0 & 9 & 21 \\
& plasmid & 166 & 3 & 108 & 22 & 33 & 0 & 82 & 9 & 75 \\
& chromosome & 890 & 16 & 37 & 540 & 297 & 18 & 31 & 407 & 434 \\
\hline \multirow{3}{*}{ Sim4 } & phage & 413 & 321 & 2 & 34 & 71 & 318 & 0 & 23 & 87 \\
& plasmid & 395 & 2 & 262 & 36 & 103 & 1 & 175 & 14 & 213 \\
& chromosome & 2,491 & 38 & 153 & 1,280 & 1012 & 50 & 114 & 970 & 1,349 \\
\hline \multirow{3}{*}{ Sim2021-Miseq } & phage & 2,014 & 503 & 2 & 45 & 1,464 & 1,068 & 17 & 65 & 864 \\
& plasmid & 3,512 & 17 & 581 & 97 & 2,817 & 19 & 689 & 126 & 2,678 \\
& chromosome & 34,211 & 172 & 182 & 6,092 & 27,765 & 272 & 613 & 9,983 & 23,343 \\
\hline \multirow{3}{*}{ Sim2021-Nano } & phage & 342 & 261 & 3 & 15 & 63 & 291 & 0 & 5 & 46 \\
& plasmid & 345 & 3 & 236 & 21 & 85 & 3 & 167 & 17 & 158 \\
& chromosome & 2,413 & 36 & 96 & 1,292 & 989 & 38 & 69 & 1,129 & 1,177 \\
\hline
\end{tabular}


bioRxiv preprint doi: https://doi.org/10.1101/2021.11.05.467408; this version posted January 26, 2022. The copyright holder for this preprint (which was not certified by peer review) is the author/funder, who has granted bioRxiv a license to display the preprint in perpetuity. It is made available under aCC-BY-ND 4.0 International license.

Table A.3. Performance of 3CAC on simulated metagenome assemblies.

\begin{tabular}{c|c|c|cccc|cccc}
\hline & True Category & \# contigs & \multicolumn{9}{c|}{ 3CAC(vV) } & \multicolumn{4}{c}{ 3CAC(PM) } \\
& & & phage plasmid chromosome & uncertain & phage plasmid chromosome uncertain \\
\hline \multirow{3}{*}{ Sim1 } & phage & 696 & 642 & 7 & 21 & 26 & 646 & 7 & 17 & 26 \\
& plasmid & 1,699 & 18 & 1,135 & 404 & 142 & 32 & 1,103 & 411 & 153 \\
& chromosome & 12,494 & 393 & 538 & 10,901 & 662 & 434 & 902 & 10,481 & 677 \\
\hline \multirow{3}{*}{ Sim2 } & phage & 2,926 & 2,728 & 9 & 77 & 112 & 2,714 & 26 & 80 & 106 \\
& plasmid & 5,350 & 50 & 2,815 & 1,440 & 1,045 & 98 & 2,871 & 1,695 & 686 \\
& chromosome & 40,412 & 915 & 1,542 & 31,712 & 6,243 & 1,278 & 3,760 & 30,817 & 4,557 \\
\hline \multirow{3}{*}{ Sim3 } & phage & 175 & 145 & 1 & 11 & 18 & 148 & 1 & 8 & 18 \\
& plasmid & 166 & 3 & 124 & 25 & 14 & 0 & 110 & 18 & 38 \\
& chromosome & 890 & 12 & 28 & 769 & 81 & 25 & 36 & 711 & 118 \\
\hline \multirow{3}{*}{ Sim4 } & phage & 413 & 336 & 2 & 35 & 40 & 328 & 0 & 27 & 58 \\
& plasmid & 395 & 2 & 306 & 41 & 46 & 4 & 210 & 43 & 138 \\
& chomosome & 2,491 & 35 & 148 & 2,087 & 221 & 71 & 143 & 1,877 & 400 \\
\hline \multirow{3}{*}{ Sim2021-Miseq } & phage & 2,014 & 1,575 & 3 & 128 & 308 & 1,662 & 24 & 153 & 175 \\
& plasmid & 3,512 & 55 & 2,596 & 472 & 389 & 66 & 2,402 & 730 & 314 \\
& chromosome & 34,211 & 674 & 1,260 & 30,076 & 2,201 & 1,008 & 2,131 & 29,364 & 1,708 \\
\hline \multirow{3}{*}{ Sim2021-Nano } & phage & 342 & 297 & 5 & 15 & 25 & 297 & 0 & 5 & 40 \\
& plasmid & 345 & 3 & 272 & 21 & 49 & 11 & 218 & 25 & 91 \\
& chromosome & 2,413 & 33 & 94 & 2,066 & 220 & 45 & 94 & 1,961 & 313 \\
\hline
\end{tabular}

Table A.4. Classification of phages, plasmids, and chromosomes on simulated and real metagenome assemblies. PPR-Meta and PPR-Meta $(0.7)$ represent running PPR-Meta on default setting and with a score threshold of 0.7 , respectively.

\begin{tabular}{|c|c|c|c|c|c|c|c|c|c|c|}
\hline & Tool & & Phage & & & Plasmid & & C & Chromoson & F1 score $(\%)$ \\
\hline & & \begin{tabular}{|c|} 
precision $(\%)$ \\
53.04
\end{tabular} & recall(\%) & F1 score(\%) & precision(\%) & ) recall(\%) & F1 score $(\%)$ & \begin{tabular}{|c|} 
precision(\%) \\
97.8
\end{tabular} & 6) recall(\%) & \begin{tabular}{|c|} 
F1 score $(\%)$ \\
34.58
\end{tabular} \\
\hline & $\begin{array}{l}\text { viralVerify } \\
3 \mathrm{CAC}(\mathrm{vV})\end{array}$ & $\begin{array}{l}53.04 \\
60.97\end{array}$ & $\begin{array}{l}40.09 \\
92.24\end{array}$ & $\begin{array}{l}45.66 \\
73.41\end{array}$ & $\begin{array}{l}61.59 \\
67.56\end{array}$ & $\begin{array}{l}22.37 \\
66.80\end{array}$ & $\begin{array}{l}32.82 \\
67.18\end{array}$ & $\begin{array}{c}97.8 \\
96.25\end{array}$ & $\begin{array}{c}21.0 \\
87.25\end{array}$ & $\begin{array}{l}34.58 \\
91.53\end{array}$ \\
\hline Sim1 & PPR-Meta(0.7) & 42.43 & 82.18 & 55.97 & 50.5 & 26.6 & 34.85 & 98.84 & 30.01 & 46.04 \\
\hline & $3 \mathrm{CAC}(\mathrm{PM})$ & 58.09 & 92.82 & 71.46 & 54.82 & 64.92 & 59.44 & 96.08 & 83.89 & 89.57 \\
\hline & PPR-Meta & 19.06 & 94.54 & 31.72 & 25.97 & 57.74 & 35.83 & 95.31 & 58.43 & 72.45 \\
\hline & viralVerify & 58.87 & 25.19 & 35.28 & 52.97 & 12.19 & 19.81 & 98.86 & 13.48 & 23.73 \\
\hline & $3 \mathrm{CAC}(\mathrm{vV})$ & 73.87 & 93.23 & 82.43 & 64.48 & 52.62 & 57.95 & 95.43 & 78.47 & 86.13 \\
\hline $\operatorname{Sim} 2$ & PPR-Meta (0.7) & 47.94 & 65.58 & 55.39 & 46.83 & 25.25 & 32.81 & 99.24 & 23.68 & 38.23 \\
\hline & $3 \mathrm{CAC}(\mathrm{PM})$ & 66.36 & 92.75 & 77.37 & 43.13 & 53.66 & 47.82 & 94.55 & 76.26 & 84.43 \\
\hline & PPR-Meta & 21.83 & 90.46 & 35.17 & 24.31 & 61.7 & 34.88 & 95.86 & 54.53 & 69.52 \\
\hline & viralVerify & 70.87 & 93.14 & 80.49 & 59.05 & 74.7 & 65.96 & 97.59 & 54.49 & 69.94 \\
\hline & $3 \mathrm{CAC}(\mathrm{vV})$ & 90.63 & 82.86 & 86.57 & 81.05 & 74.70 & 77.74 & 95.53 & 86.40 & 90.74 \\
\hline Sim3 & PPR-Meta (0.7) & 74.55 & 93.71 & 83.04 & 58.67 & 53.01 & 55.70 & 98.65 & 41.12 & 58.05 \\
\hline & $3 \mathrm{CAC}(\mathrm{PM})$ & 85.55 & 84.57 & 85.06 & 74.83 & 66.27 & 70.29 & 96.47 & 79.89 & 87.40 \\
\hline & PPR-Meta & 60.63 & 99.43 & 75.33 & 42.04 & 84.34 & 56.11 & 97.38 & 66.85 & 79.28 \\
\hline & viralVerify & 75.81 & 91.04 & 82.73 & 46.88 & 76.2 & 58.05 & 98.64 & 46.61 & 63.30 \\
\hline & $3 \mathrm{CAC}(\mathrm{vV})$ & 90.08 & 81.36 & 85.50 & 67.70 & 77.47 & 72.26 & 96.49 & 83.90 & 89.76 \\
\hline Sim 4 & PPR-Meta (0.7) & 71.54 & 87.65 & 78.78 & 50.0 & 49.87 & 49.94 & 99.55 & 35.69 & 52.54 \\
\hline & $3 \mathrm{CAC}(\mathrm{PM})$ & 81.39 & 79.42 & 80.39 & 59.49 & 53.16 & 56.15 & 96.40 & 75.35 & 84.59 \\
\hline & PPR-Meta & 60.3 & 98.55 & 74.82 & 33.17 & 84.05 & 47.57 & 97.47 & 63.51 & 76.91 \\
\hline & viralVerify & 52.49 & 29.34 & 37.64 & 60.57 & 21.87 & 32.13 & 99.01 & 16.71 & 28.60 \\
\hline & 3CAC(vV) & 68.36 & 78.20 & 72.95 & 67.27 & 73.92 & 70.44 & 98.04 & 87.91 & 92.70 \\
\hline Sim2021-Miseq & PPR-Meta (0.7) & 39.24 & 72.24 & 50.86 & 47.98 & 28.13 & 35.47 & 99.14 & 27.91 & 43.55 \\
\hline & 3CAC $(\mathrm{PM})$ & 60.75 & 82.52 & 69.98 & 52.71 & 68.39 & 59.54 & 97.08 & 85.83 & 91.11 \\
\hline & PPR-Meta & 17.16 & 85.55 & 28.59 & 23.43 & 63.30 & 34.20 & 96.62 & 57.08 & 71.76 \\
\hline & viralVerify & 64.73 & 84.80 & 73.42 & 55.69 & 79.42 & 65.47 & 99.48 & 47.99 & 64.75 \\
\hline & $3 \mathrm{CAC}(\mathrm{vV})$ & 89.19 & 86.84 & 88.00 & 73.32 & 78.84 & 75.98 & 98.29 & 85.62 & 91.52 \\
\hline Sim2021-Nano & PPR-Meta (0.7) & 66.23 & 89.47 & 76.12 & 62.22 & 56.81 & 59.39 & 99.71 & 43.31 & 60.39 \\
\hline & $3 \mathrm{CAC}(\mathrm{PM})$ & 84.14 & 86.84 & 85.47 & 69.87 & 63.19 & 66.36 & 98.49 & 81.27 & 89.06 \\
\hline & PPR-Meta & 36.42 & 98.28 & 53.15 & 29.39 & 87.85 & 44.04 & 99.46 & 72.45 & 83.84 \\
\hline & viralVerify & 8.65 & 8.88 & 8.76 & 7.13 & 3.77 & 4.93 & 99.78 & 5.15 & 9.79 \\
\hline & $3 \mathrm{CAC}(\mathrm{vV})$ & 11.97 & 40.21 & 18.44 & 2.66 & 10.52 & 4.25 & 99.49 & 33.62 & 50.26 \\
\hline Gut-Hiseq & PPR-Meta $(0.7)$ & 2.24 & 55.09 & 4.30 & 3.50 & 19.38 & 5.93 & 99.81 & 26.78 & 42.23 \\
\hline & $3 \mathrm{CAC}(\mathrm{PM})$ & 7.02 & 58.22 & 12.53 & 3.75 & 16.39 & 6.11 & 99.56 & 52.15 & 68.45 \\
\hline & PPR-Meta & 1.13 & 80.94 & 2.23 & 1.51 & 49.94 & 2.94 & 99.62 & 56.87 & 72.41 \\
\hline & viralVerify & 3.80 & 37.50 & 6.90 & 6.54 & 32.81 & 10.91 & 100 & 44.02 & 61.13 \\
\hline & $3 \mathrm{CAC}(\mathrm{vV})$ & 27.59 & 100 & 43.24 & 17.65 & 23.44 & 20.13 & 99.74 & 82.89 & 90.54 \\
\hline Gut-Pacbio & PPR-Meta $(0.7)$ & 8.25 & 100 & 15.24 & 19.55 & 40.63 & 26.40 & 99.63 & 39.94 & 57.02 \\
\hline & $3 \mathrm{CAC}(\mathrm{PM})$ & 10.00 & 87.50 & 17.95 & 24.14 & 32.81 & 27.81 & 99.65 & 79.49 & 88.43 \\
\hline & PPR-Meta & 2.47 & 100 & 4.82 & 4.95 & 67.19 & 9.22 & 99.55 & 75.68 & 85.99 \\
\hline
\end{tabular}


bioRxiv preprint doi: https://doi.org/10.1101/2021.11.05.467408; this version posted January 26, 2022. The copyright holder for this preprint (which was not certified by peer review) is the author/funder, who has granted bioRxiv a license to display the preprint in perpetuity. It is made available under aCC-BY-ND 4.0 International license.

Table A.5. Performance of three-class classifiers on simulated and real metagenome assemblies. PPR-Meta and PPR-Meta(0.7) represent running PPRMeta on default setting and with a score threshold of 0.7 , respectively.

\begin{tabular}{|c|c|c|c|c|c|c|}
\hline Dataset & Evaluation Criteria & viralVerify & $3 \mathrm{CAC}(\mathrm{vV})$ & PPR-Meta(0.7) & 3CAC(PM) & PPR-Meta \\
\hline \multirow{3}{*}{ Sim1 } & Precision & $85.81 \%$ & $90.18 \%$ & $79.08 \%$ & $87.15 \%$ & $60.04 \%$ \\
\hline & Recall & $22.05 \%$ & $85.15 \%$ & $32.06 \%$ & $82.14 \%$ & $60.04 \%$ \\
\hline & F1 score & $35.08 \%$ & $87.59 \%$ & $45.62 \%$ & $84.57 \%$ & $60.04 \%$ \\
\hline \multirow{3}{*}{ Sim2 } & Precision & $85.53 \%$ & $90.23 \%$ & $77.67 \%$ & $83.99 \%$ & $57.47 \%$ \\
\hline & Recall & $14.04 \%$ & $76.52 \%$ & $26.37 \%$ & $74.77 \%$ & $57.47 \%$ \\
\hline & F1 score & $24.12 \%$ & $82.81 \%$ & $39.37 \%$ & $79.11 \%$ & $57.47 \%$ \\
\hline \multirow{3}{*}{ Sim3 } & Precision & $82.39 \%$ & $92.84 \%$ & $83.4 \%$ & $91.67 \%$ & $73.84 \%$ \\
\hline & Recall & $62.71 \%$ & $84.32 \%$ & $50.2 \%$ & $78.72 \%$ & $73.84 \%$ \\
\hline & F1 score & $71.22 \%$ & $88.38 \%$ & $62.68 \%$ & $84.70 \%$ & $73.84 \%$ \\
\hline \multirow{3}{*}{$\operatorname{Sim} 4$} & Precision & $79.4 \%$ & $91.34 \%$ & $80.76 \%$ & $89.35 \%$ & $70.35 \%$ \\
\hline & Recall & $55.71 \%$ & $82.81 \%$ & $43.89 \%$ & $73.20 \%$ & $70.35 \%$ \\
\hline & F1 score & $65.48 \%$ & $86.87 \%$ & $56.87 \%$ & $80.47 \%$ & $70.35 \%$ \\
\hline \multirow{3}{*}{ Sim2021-Miseq } & Precision & $86.63 \%$ & $92.96 \%$ & $77.87 \%$ & $89.05 \%$ & $59.07 \%$ \\
\hline & Recall & $17.81 \%$ & $86.18 \%$ & $30.17 \%$ & $84.12 \%$ & $59.07 \%$ \\
\hline & F1 score & $29.55 \%$ & $89.45 \%$ & $43.49 \%$ & $86.51 \%$ & $59.07 \%$ \\
\hline \multirow{3}{*}{ Sim2021-Nano } & Precision & $81.84 \%$ & $93.91 \%$ & $84.77 \%$ & $93.22 \%$ & $74.91 \%$ \\
\hline & Recall & $55.55 \%$ & $85.00 \%$ & $49.90 \%$ & $79.87 \%$ & $74.91 \%$ \\
\hline & F1 score & $66.18 \%$ & $89.23 \%$ & $62.82 \%$ & $86.03 \%$ & $74.91 \%$ \\
\hline \multirow{3}{*}{ Gut-Hiseq } & Precision & $89.24 \%$ & $90.12 \%$ & $71.41 \%$ & $90.64 \%$ & $56.90 \%$ \\
\hline & Recall & $5.15 \%$ & $33.48 \%$ & $26.81 \%$ & $51.92 \%$ & $56.90 \%$ \\
\hline & F1 score & $9.74 \%$ & $48.83 \%$ & $38.98 \%$ & $66.02 \%$ & $56.90 \%$ \\
\hline \multirow{3}{*}{ Gut-Pacbio } & Precision & $84.70 \%$ & $97.47 \%$ & $90.35 \%$ & $96.35 \%$ & $75.61 \%$ \\
\hline & Recall & $43.86 \%$ & $82.12 \%$ & $40.05 \%$ & $78.87 \%$ & $75.61 \%$ \\
\hline & F1 score & $57.79 \%$ & $89.14 \%$ & $55.50 \%$ & $86.74 \%$ & $75.61 \%$ \\
\hline
\end{tabular}

Table A.6. Running time in minutes of the classifiers. Row 3CAC shows the running time for the second phase of 3CAC algorithm.

\begin{tabular}{l|c|c|c|c|c|c|c|c}
\hline & Sim1 & Sim2 & Sim3 & Sim4 & $\begin{array}{c}\text { Sim2021 } \\
\text {-Miseq }\end{array}$ & $\begin{array}{c}\text { Sim2021 } \\
\text {-Nano }\end{array}$ & Gut-HiSeq & Gut-Pacbio \\
\hline viralVerify & 141 & 201 & 165 & 218 & 314 & 324 & 238 & 292 \\
\hline PPR-Meta & 22 & 32 & 23 & 33 & 69 & 65 & 67 & 50 \\
\hline PlasClass & 0.43 & 0.61 & 0.72 & 0.72 & 0.6 & 0.68 & 0.7 & 0.22 \\
\hline DeepVirFinder & 2.5 & 5.5 & 2 & 3.5 & 6.3 & 3.4 & 32 & 1 \\
\hline 3CAC & 1.5 & 3.5 & 0.5 & 0.5 & 2.1 & 0.5 & 4 & 55 \\
\hline
\end{tabular}


Table A.7. Performance of 3CAC with different contig scanning orders. In the correction step, each set of incongruous contigs with the same number of classified neighbors was implemented by a queue data structure. The same was done for implied contigs in the propagation step. These queues determine the contig scanning order. To test the effect of the order, we modified 3CAC to use lists instead of queues, and inserted any new contig to a random location in the corresponding list. The table shows the results for the original $3 \mathrm{CAC}(\mathrm{O})$ and for two random orders $\mathrm{R} 1$ and $\mathrm{R} 2$.

\begin{tabular}{|c|c|c|c|c|c|c|c|}
\hline \multirow[t]{2}{*}{ Dataset } & Evaluation & \multicolumn{3}{|c|}{ 3CAC (vV) } & \multicolumn{3}{|c|}{ 3CAC(PM) } \\
\hline & Criteria & $\mathrm{O}$ & R1 & R2 & $\mathrm{O}$ & R1 & R2 \\
\hline \multirow{3}{*}{ Sim1 } & Precision & $90.18 \%$ & $89.70 \%$ & $89.49 \%$ & $87.15 \%$ & $86.71 \%$ & $86.65 \%$ \\
\hline & Recall & $85.15 \%$ & $84.96 \%$ & $84.79 \%$ & $82.14 \%$ & $82.05 \%$ & $82.11 \%$ \\
\hline & F1 score & $87.59 \%$ & $87.27 \%$ & $87.08 \%$ & $84.57 \%$ & $84.31 \%$ & $84.32 \%$ \\
\hline \multirow{3}{*}{ Sim2 } & Precision & $90.23 \%$ & $90.19 \%$ & $89.76 \%$ & $83.99 \%$ & $83.72 \%$ & $83.71 \%$ \\
\hline & Recall & $76.52 \%$ & $76.70 \%$ & $76.32 \%$ & $74.77 \%$ & $74.76 \%$ & $74.73 \%$ \\
\hline & F1 score & $82.81 \%$ & $82.90 \%$ & $82.50 \%$ & $79.11 \%$ & $78.99 \%$ & $78.96 \%$ \\
\hline \multirow{3}{*}{ Sim3 } & Precision & $92.84 \%$ & $92.68 \%$ & $92.67 \%$ & $91.67 \%$ & $90.52 \%$ & $91.52 \%$ \\
\hline & Recall & $84.32 \%$ & $84.32 \%$ & $84.24 \%$ & $78.72 \%$ & $78.31 \%$ & $78.88 \%$ \\
\hline & F1 score & $88.38 \%$ & $88.30 \%$ & $88.26 \%$ & $84.70 \%$ & $83.97 \%$ & $84.73 \%$ \\
\hline \multirow{3}{*}{ Sim4 } & Precision & $91.34 \%$ & $91.09 \%$ & $90.98 \%$ & $89.35 \%$ & $89.65 \%$ & $89.32 \%$ \\
\hline & Recall & $82.81 \%$ & $82.72 \%$ & $82.60 \%$ & $73.20 \%$ & $73.81 \%$ & $73.54 \%$ \\
\hline & F1 score & 86.87 & 86.70 & $86.59 \%$ & $80.47 \%$ & $80.96 \%$ & $80.67 \%$ \\
\hline \multirow{3}{*}{ Sim2021-Miseq } & Precision & $92.96 \%$ & $92.92 \%$ & $92.55 \%$ & $89.05 \%$ & $88.94 \%$ & $88.73 \%$ \\
\hline & Recall & $86.18 \%$ & $86.44 \%$ & $86.09 \%$ & $84.12 \%$ & $84.24 \%$ & $84.05 \%$ \\
\hline & F1 score & $89.45 \%$ & $89.56 \%$ & $89.21 \%$ & $86.51 \%$ & $86.53 \%$ & $86.32 \%$ \\
\hline \multirow{3}{*}{ Sim2021-Nano } & Precision & $93.91 \%$ & $93.91 \%$ & $93.67 \%$ & $93.22 \%$ & $92.66 \%$ & $92.43 \%$ \\
\hline & Recall & $85.00 \%$ & $85.13 \%$ & $84.90 \%$ & $79.87 \%$ & $79.45 \%$ & $79.55 \%$ \\
\hline & F1 score & $89.23 \%$ & $89.31 \%$ & $89.07 \%$ & $86.03 \%$ & $85.55 \%$ & $85.51 \%$ \\
\hline \multirow{3}{*}{ Gut-Hiseq } & Precision & $90.12 \%$ & $89.86 \%$ & $89.73 \%$ & $90.64 \%$ & $90.50 \%$ & $90.35 \%$ \\
\hline & Recall & $33.48 \%$ & $33.42 \%$ & $33.37 \%$ & $51.92 \%$ & $52.00 \%$ & $51.93 \%$ \\
\hline & F1 score & $48.83 \%$ & $48.72 \%$ & $48.65 \%$ & $66.02 \%$ & $66.05 \%$ & $65.95 \%$ \\
\hline \multirow{3}{*}{ Gut-Pacbio } & Precision & $97.47 \%$ & $97.53 \%$ & $97.58 \%$ & $96.35 \%$ & $96.10 \%$ & $96.33 \%$ \\
\hline & & $82.12 \%$ & $82.24 \%$ & $82.35 \%$ & $78.87 \%$ & $78.83 \%$ & $79.00 \%$ \\
\hline & F1 score & $89.14 \%$ & 8022 & $89.32 \%$ & $86.74 \%$ & $86.61 \%$ & $86.81 \%$ \\
\hline
\end{tabular}


Table A.8. Comparison of PlasClass and 3CAC on plasmid classification in simulated metagenome assemblies. PlasClass (0.5) and PlasClass $(0.7)$ classified a contig as plasmid if its score $\geq 0.7$ and $>0.5$, respectively. Here, precision was calculated as the fraction of correctly classified plasmid contigs among all contigs classified as plasmids, and the recall was calculated as the fraction of correctly classified plasmid contigs among all plasmid contigs.

\begin{tabular}{|c|c|c|c|c|c|}
\hline Dataset & Evaluation Criteria & PlasClass(0.5) & PlasClass(0.7) & 3CAC(vV) & 3CAC(PM) \\
\hline \multirow{3}{*}{ Sim1 } & Precision & $20.32 \%$ & $21.48 \%$ & $67.56 \%$ & $54.82 \%$ \\
\hline & Recall & $68.16 \%$ & $57.27 \%$ & $66.80 \%$ & $64.92 \%$ \\
\hline & F1 score & $31.30 \%$ & $31.25 \%$ & $67.18 \%$ & $59.44 \%$ \\
\hline \multirow{3}{*}{ Sim2 } & Precision & $17.49 \%$ & $19.04 \%$ & $64.48 \%$ & $43.13 \%$ \\
\hline & Recall & $68.41 \%$ & $57.42 \%$ & $52.62 \%$ & $53.66 \%$ \\
\hline & F1 score & $27.86 \%$ & $28.60 \%$ & $57.95 \%$ & $47.82 \%$ \\
\hline \multirow{3}{*}{ Sim3 } & Precision & $31.20 \%$ & $42.41 \%$ & $81.05 \%$ & $74.83 \%$ \\
\hline & Recall & $87.95 \%$ & $82.53 \%$ & $74.70 \%$ & $66.27 \%$ \\
\hline & F1 score & $46.06 \%$ & $56.03 \%$ & $77.74 \%$ & $70.29 \%$ \\
\hline \multirow{3}{*}{ Sim4 } & Precision & $27.32 \%$ & $35.00 \%$ & $67.11 \%$ & $59.49 \%$ \\
\hline & Recall & $89.37 \%$ & $77.97 \%$ & $77.47 \%$ & $53.16 \%$ \\
\hline & F1 score & $41.85 \%$ & $48.31 \%$ & $71.92 \%$ & $56.15 \%$ \\
\hline \multirow{3}{*}{ Sim2021-Miseq } & Precision & $15.67 \%$ & $16.56 \%$ & $67.27 \%$ & $52.71 \%$ \\
\hline & Recall & $71.50 \%$ & $58.66 \%$ & $73.92 \%$ & $68.39 \%$ \\
\hline & F1 score & $25.71 \%$ & $25.82 \%$ & $70.44 \%$ & $59.54 \%$ \\
\hline \multirow{3}{*}{ Sim2021-Nano } & Precision & $26.98 \%$ & $36.40 \%$ & $73.32 \%$ & $69.87 \%$ \\
\hline & Recall & $86.96 \%$ & $81.45 \%$ & $78.84 \%$ & $63.19 \%$ \\
\hline & F1 score & $41.18 \%$ & $50.31 \%$ & $75.98 \%$ & $66.36 \%$ \\
\hline
\end{tabular}

Table A.9. Comparison of DeepVirFinder and 3CAC on phage classification in simulated metagenome assemblies. DeepVirFinder(0.05) and DeepVirFinder(0.03) classified a contig as phage if its pvalue $\leq 0.05$ and $\leq 0.03$, respectively. Here, precision was calculated as the fraction of correctly classified phage contigs among all contigs classified as phages, and the recall was calculated as the fraction of correctly classified phage contigs among all phage contigs. 76 contigs longer than $2 \mathrm{Mb}$ were excluded from the comparison since DeepVirFinder did not terminate successfully on these long contigs.

\begin{tabular}{|c|c|c|c|c|c|}
\hline Dataset & Evaluation Criteria & DeepVirFinder(0.05) & DeepVirFinder(0.03) & $3 \mathrm{CAC}(\mathrm{vV})$ & 3CAC(PM) \\
\hline \multirow{3}{*}{ Sim1 } & Precision & $29.46 \%$ & $39.10 \%$ & $60.97 \%$ & $58.09 \%$ \\
\hline & Recall & $83.76 \%$ & $77.59 \%$ & $92.24 \%$ & $92.82 \%$ \\
\hline & F1 score & $43.59 \%$ & $52.00 \%$ & $73.41 \%$ & $71.46 \%$ \\
\hline \multirow{3}{*}{$\operatorname{Sim} 2$} & Precision & $30.99 \%$ & $39.52 \%$ & $73.87 \%$ & $66.36 \%$ \\
\hline & Recall & $70.95 \%$ & $63.81 \%$ & $93.23 \%$ & $92.75 \%$ \\
\hline & F1 score & $43.14 \%$ & $48.81 \%$ & $82.43 \%$ & $77.37 \%$ \\
\hline \multirow{3}{*}{ Sim3 } & Precision & $49.09 \%$ & $60.98 \%$ & $90.63 \%$ & $85.55 \%$ \\
\hline & Recall & $92.00 \%$ & $85.71 \%$ & $82.86 \%$ & $84.57 \%$ \\
\hline & F1 score & $64.02 \%$ & $71.26 \%$ & $86.57 \%$ & $85.06 \%$ \\
\hline \multirow{3}{*}{ Sim4 } & Precision & $46.22 \%$ & $58.63 \%$ & $90.08 \%$ & $81.39 \%$ \\
\hline & Recall & $90.31 \%$ & $83.05 \%$ & $81.36 \%$ & $79.42 \%$ \\
\hline & F1 score & $61.15 \%$ & $68.74 \%$ & $85.50 \%$ & $80.39 \%$ \\
\hline \multirow{3}{*}{ Sim2021-Miseq } & Precision & $28.52 \%$ & $37.34 \%$ & $68.36 \%$ & $60.75 \%$ \\
\hline & Recall & $74.48 \%$ & $67.03 \%$ & $78.20 \%$ & $82.52 \%$ \\
\hline & F1 score & $47.97 \%$ & $41.24 \%$ & $72.95 \%$ & $69.98 \%$ \\
\hline \multirow{3}{*}{ Sim2021-Nano } & Precision & $37.63 \%$ & $55.70 \%$ & $89.19 \%$ & $84.14 \%$ \\
\hline & Recall & $94.74 \%$ & $90.06 \%$ & $86.84 \%$ & $86.84 \%$ \\
\hline & F1 score & $53.87 \%$ & $68.83 \%$ & $88.00 \%$ & $85.47 \%$ \\
\hline
\end{tabular}




\section{B Supplementary figures}
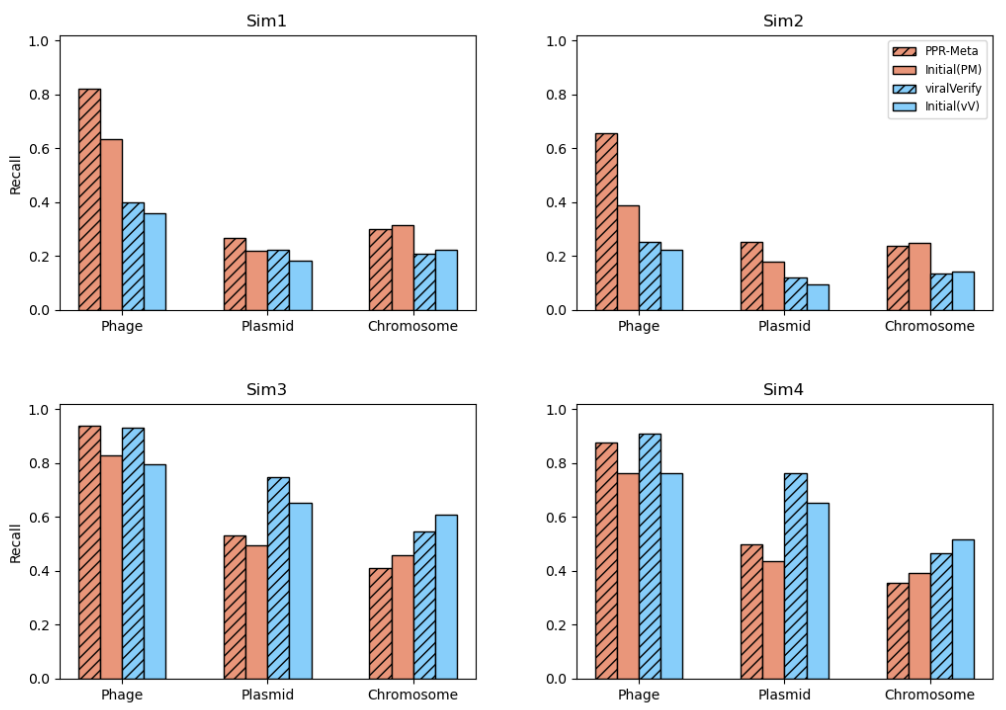

Fig. B.1. Recall of the initial classification of 3CAC compared to PPR-Meta and viralVerify. 

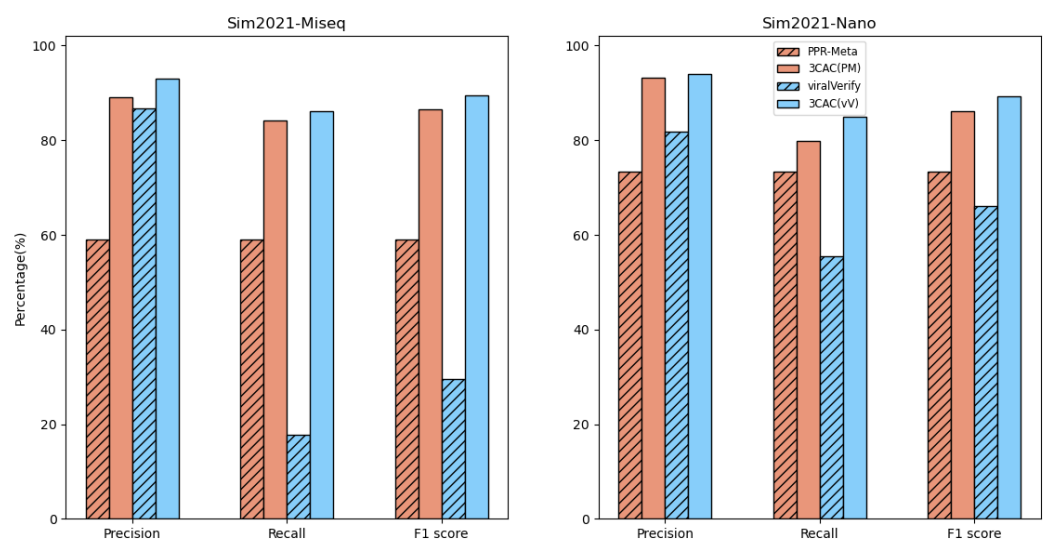

Fig. B.2. Performance of three-class classifiers on metagenomes simulated from newly released genomes. 